\title{
Review
}

Acta Haematol 2020;143:112-117

DOI: $10.1159 / 000500315$
Received: January 12, 2019

Accepted after revision: April 10, 2019

Published online: July 9, 2019

\section{The Role of Tumor-Associated Macrophages in Leukemia}

\author{
Yueyang $\mathrm{Li}^{\mathrm{a}}$ M. James You ${ }^{\mathrm{b}}$ Yaling Yang ${ }^{\mathrm{b}}$ Dongzhi Hu${ }^{\mathrm{a}}$ Chen Tian ${ }^{\mathrm{a}}$ \\ a Department of Hematology, Tianjin Medical University Cancer Institute and Hospital, National Clinical \\ Research Center for Cancer, Key Laboratory of Cancer Prevention and Therapy, Tianjin, China; \\ ${ }^{b}$ Department of Hematopathology, The University of Texas MD Anderson Cancer Center, Houston, TX, USA
}

\section{Keywords}

Tumor-associated macrophages · Leukemia-associated macrophages · Nurse-like cells · Polarization · Leukemic cell growth

\begin{abstract}
In addition to intrinsic factors, leukemia cell growth is influenced by the surrounding nonhematopoietic cells in the leukemic microenvironment, including fibroblasts, mesenchymal stem cells, vascular cells, and various immune cells. Despite the fact that macrophages are an important component of human innate immunity, tumor-associated macrophages (TAMs) have long been considered as an accomplice promoting tumor growth and metastasis. TAMs are activated by an abnormal malignant microenvironment, polarizing into a specific phenotype and participating in tumor progression. TAMs that exist in the microenvironment of different types of leukemia are called leukemia-associated macrophages (LAMs), which are reported to be associated with the progression of leukemia. This review describes the role of LAMs in different leukemia subtypes.

(c) 2019 S. Karger AG, Basel
\end{abstract}

Y. Li and M.J. You contributed equally to this review.

\section{Background}

Macrophages that infiltrate into tumor tissues are regarded as tumor-associated macrophages (TAMs), which are closely involved in tumorigenesis [1-3]. TAMs contribute to tumor progression from different aspects, including promoting genetic instability, nurturing cancer stem cells, paving the way to metastasis, and taming protective adaptive immunity. The correlation between TAMs and the prognosis of patients has been revealed by previous studies [4]. Cai et al. [5] reported that in pancreatic neuroendocrine tumors, TAM infiltration was associated with a worse disease-free survival. TAM infiltration was the sole independent predictor of disease-free survival for patients with pancreatic neuroendocrine tumors $(p=0.02)$. TAMs infiltrated into liver metastasis conferred a poor prognosis in human colorectal cancer [6].

Mounting evidence has demonstrated that leukemia cells interact with the bone marrow microenvironment, which is composed of many types of cell, such as osteoblasts, osteoclasts, stromal cells, vascular endothelial cells, and immune cells, including natural killer cells and leukemia-associated macrophages (LAMs) [7-9]. The immunosuppressive microenvironment formed by leuke-

Chen Tian

Department of Hematology, Tianjin Medical University Cancer Institute and Hospital National Clinical Research Center for Cancer

Key Laboratory of Cancer Prevention and Therapy, Tianjin 300011 (China)

E-Mail tcgirl2001@sina.com 


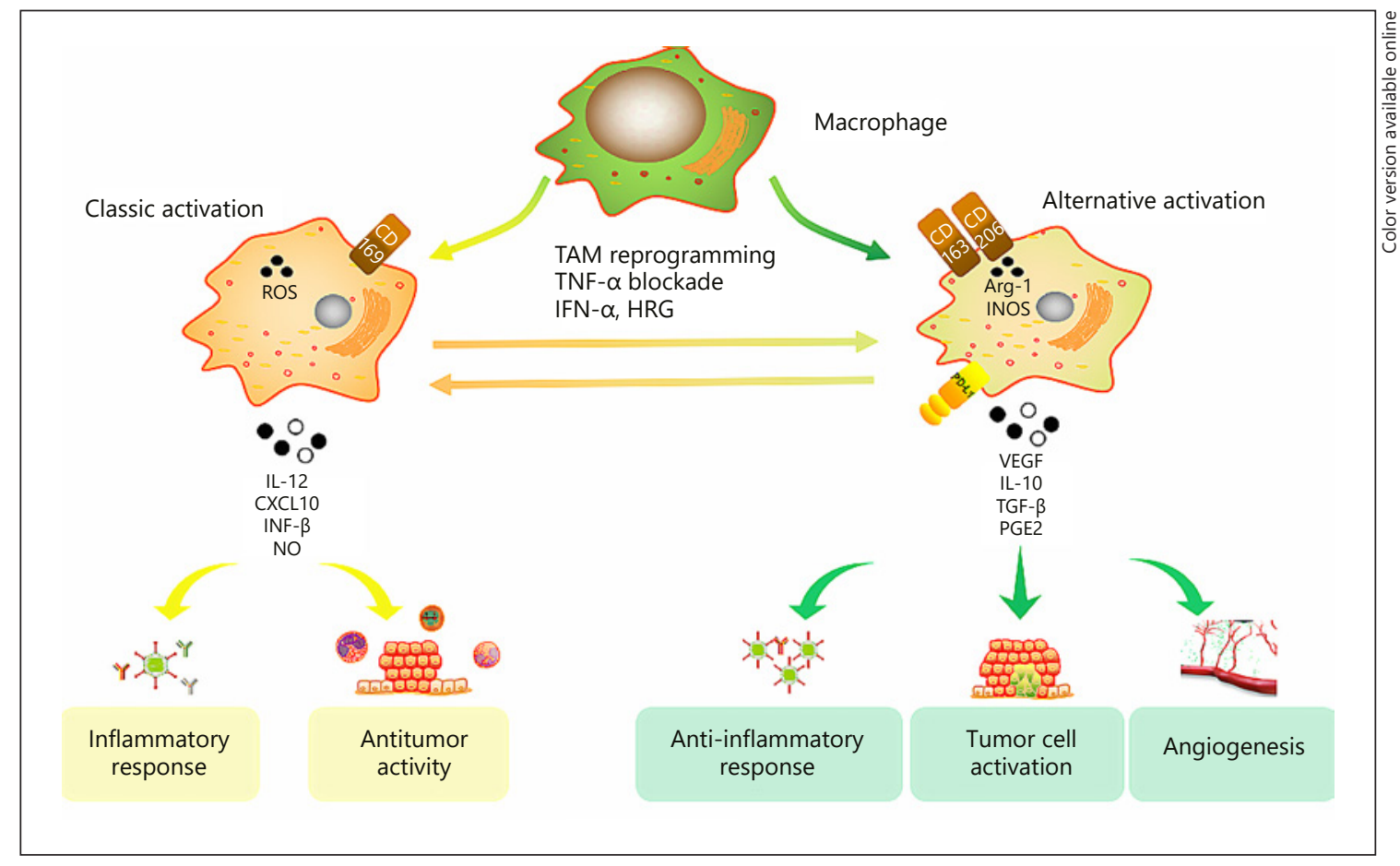

Fig. 1. Polarization of macrophages in leukemia. Leukemia-associated macrophages differentiated into two types: classically activated (M1) macrophages and alternatively activated (M2) macrophages under a leukemic microenvironment. TAM, tumor-associated macrophage.

mia cells reprogram the phenotypes and functions of macrophages through various pathways, leading to a decreased ability of macrophages to recognize and phagocytize antigens which result in immune escape [8].

\section{Heterogeneity of Macrophages and Their Polarization}

Macrophages are highly heterogeneous mainly in morphology, biochemistry, phenotypes, and functions [9-11]. TAMs were once thought to have antitumor activity. However, recent studies have confirmed that tumor cells induce TAMs to secrete assorted factors and cytokines to promote tumor cell growth instead of stimulating the immune response to antitumor development [12].

The process in which macrophages interact with external stimulus signals and differentiate into subpopulations to perform different functions is called polarization [13], which is the result of a complex bidirectional interaction between the tumor microenvironment and macrophages. Macrophages could develop into classically activated (M1) or alternatively activated (M2) macrophages
(Fig. 1). M1 macrophages, participating in immune defense against external pathogenic microorganisms and promoting antitumor immunity, are triggered by lipopolysaccharide, IFN- $\gamma$, TNF- $\alpha$, and granulocyte-macrophage colony-stimulating factor [14-16]. M2 macrophages, also known as selectively activated macrophages [17], are stimulated by interleukin (IL)-4, IL-10, IL-13, secreting arginase, metalloproteinase, and transforming growth factor- $\beta$ [18].

In fact, M2 macrophages generally include all activated macrophages except M1 macrophages and can be further divided into 4 subtypes: M2a, M2b, M2c, and M2d. M2 macrophages are believed to be associated with tumor promotion by inducing angiogenesis, immunosuppression, and tumor cell metastasis. M2 macrophages highly express CD163 (hemoglobin scavenger receptor), CD204 (class A macrophage scavenger receptor), and CD206 (mannose receptor, $\mathrm{C}$ type 1 ), which are widely used as markers for M2 macrophages [19-21]. However, CD163 expression is upregulated by IL-10 and corticosteroids, but not by IL-4 [21]. CD206 expression is upregulated by IL-4, but not by IL-10 [22]. This discrepancy suggests that differentiation of macrophages is heterogeneous and seems to be ambiguous. 


\section{The Role of LAMs in Different Subtypes of Leukemia}

The functional and phenotypic characteristics of LAMs and TAMs are different according to organ-specific microenvironments. In recent years, the role of LAMs in leukemia has been investigated especially in acute lymphoblastic leukemia (ALL), acute myeloid leukemia (AML), and chronic lymphocytic leukemia (CLL) [2330].

\section{ALL-Associated Macrophages}

In a Notch1 overexpression-induced mouse model of acute T-lymphocytic leukemia (T-ALL), the gene expression profiles of LAMs originating from bone marrow and the spleen were significantly different. Besides, proliferation of T-ALL cells was triggered more potently by splenic LAMs than by bone marrow LAMs after coculture, suggesting distinct effects of different microenvironments on LAMs [23]. In the same model, hepatic LAMs exhibited a more M1-like phenotype by expressing increased levels of cytokines recruiting inflammatory cells, resulting in a proinflammatory hepatic microenvironment, which was distinct from that of TAMs in hepatocellular carcinoma [24]. In addition, peritoneal macrophages and peritoneal LAMs were shown to simultaneously express high levels of iNOS and Arg1, and lower levels of CSF-1, TGF- $\beta_{1}$, and VEGF-A, which is in contrast to what is typically seen in macrophages of other origin. These results revealed the diverse functional and phenotypic characteristics of LAMs originating from different organs [25].

Komohara et al. [26] analyzed 58 T-ALL patients' samples using CD68 (a pan-macrophage marker) and CD163 (an M2 marker) immunostaining, demonstrating that high numbers of CD68+ and CD163+ cells were associated with poor prognosis and that the percentage of CD163+ cells was an independent prognostic factor by multivariate analysis. In vitro experiments showed that the proliferation of T-ALL cell lines was significantly increased after coculturing with M2 macrophages due to the secretion of C5a, TNF- $\alpha$, growth-related oncogene- $\alpha$, CCL1/I-309, and IL-6 [26]. Macrophage colony-stimulating factor (M-CSF) is an important cytokine for macrophage differentiation. There are two major alternative splicing isoforms: secretory M-CSF and membranebound M-CSF. Though both isoforms induce macrophages to differentiate into the M2 type, membranebound M-CSF and secretory M-CSF induce different spectrums of phenotype-associated genes in LAMs [27].

Recently, Komohara et al. [28] reported that the MCSF receptor inhibitor could play a role in the antilym- phoma effect through suppressing macrophages stimulated with a conditioned medium from T-ALL cells. These results suggest that interference with interactions between M2 macrophages and leukemia cells may have therapeutic potential for patients with T-ALL.

\section{AML-Associated Macrophages}

It was proved that LAMs play an important role in the survival and drug resistance of AML cells [29]. It was also confirmed that LAMs could protect AML cells from apoptosis induced by cytarabine. Therefore, exploring therapeutic strategies for LAMs may help reverse drug resistance in AML cells. However, the detailed molecular mechanisms remain to be identified.

AI-Matary et al. [29] have recently made a breakthrough in understanding the protection of AML cells by LAMs. They found that AML cells lead to the invasion of LAMs into the bone marrow and spleen of leukemic patients and mice. In different gene mutation-induced AML mouse models, LAMs supported the in vitro expansion of AML cell lines better than macrophages from nonleukemic mice, and LAM infiltration correlated with the survival of the mice. The absence of growth factor independent 1 transcriptional repressor could reprogram LAMs polarized towards an antitumor state both in vitro and in vivo.

Similarly, heterogeneity in LAM activation in tissuespecific leukemic microenvironments was also observed in AML models. Splenic LAMs evolved with more M2 characteristics, while bone marrow LAMs differentiated with more M1 characteristics in an MLL-AF9 AML mouse model. Furthermore, IRF7 contributed to M2 characteristic polarization through activation of the SAPK/JNK pathway. Targeting the IRF7-SAPK/JNK pathway to induce M1 characteristics in LAMs led to prolonged survival of AML mice [30].

Other intriguing therapeutic approaches involving macrophages in AML that deserve to be explored are antibodies involving the CD47 molecule. CD47 is a transmembrane protein and inhibits phagocytosis by binding to its receptor signal-regulatory protein- $\alpha$, which is expressed on phagocytes. Absence of CD47 on hematopoietic cells led to rapid clearance of these cells by macrophages. Abundant CD47 expression has been observed in hematopoietic malignancies. Monoclonal antibodies against human CD47 have been demonstrated to have efficacy in AML [31]. Anti-CD47 antibody-based approaches enable phagocytosis of AML cells by macrophages. 
Recently, a new lysine acetyltransferase named monocytic leukemia zinc finger protein (MOZ) was found to form fusion proteins with many regulators in AML. A low level of MOZ was associated with poor prognosis. Genetic silencing of MOZ resulted in diminished M1 activation of macrophages. MOZ contributed to the macrophage differentiation and polarization program, which was deregulated in AML [32].

\section{CLL-Associated Macrophages}

In 2000, Burger et al. [33] found that some large, adherent cells differentiated from the peripheral mononuclear cells of CLL patients could promote the survival of CLL cells in vitro, and the authors named them nurse-like cells (NLCs). Afterwards, Tsukada et al. [34] demonstrated that NLCs in CLL patients were a component of the leukemic microenvironment which corresponded to TAMs in solid tumors. In 2015, Boissard et al. [35] once again confirmed that NLCs are CLL-associated macrophages. NLCs were revealed to present an M2 phenotype characterized by high expression of CD11b, CD163, CD206, HLA-DR, HGF, and IDO [35-37]. NLCs could support the survival and proliferation of CLL cells and protect CLL cells from drug-induced apoptosis; however, the mechanism has not been fully clarified. Recently, macrophage depletion was demonstrated as a promising strategy for sensitizing CLL cells to apoptosis and inhibiting disease progression in a mouse model.

It was reported that NLCs may promote the proliferation of CLL cells through the chemokines CXCL12 and CXCL13 and vascular cell adhesion molecule 1. Boissard et al. [35] found that the interaction between lymphocyte function-associated antigen 3 (LFA-3) expressed in CLL at significantly higher levels and CD2 expressed on NLCs was important for CLL growth. In an exploratory cohort of 60 CLL patients receiving frontline immunochemotherapy, increased levels of soluble LFA-3 were found to correlate with shorter overall survival.

The role of the monocyte/macrophage lineage in CLL has been extensively studied in vitro, but only recently has been investigated in in vivo models [36]. It has been found that genetic inactivation of migration inhibitory factor in the E $\mu$-TCL1 mouse model of CLL could delay the development of leukemia. Migration inhibitory factor seems to act as a cofactor for the pathogenesis of CLL by favoring the accumulation of macrophages in CLL-invaded organs $[37,38]$.

Galletti et al. [39] found that macrophages and leukemic cells crosstalk during CLL progression. Absence of macrophages could impair CLL engraftment, drastically reduced leukemic cell growth, and favorably prolonged the survival of mouse models. The CSF 1 receptor (CSF1R) and TNF pathways were two major methods of targeting macrophages. Blockade of these two signaling pathways led to macrophage killing, which reprogramed the tumor microenvironment toward an antitumoral phenotype. IFN- $\gamma$ is reported to promote the polarization of macrophages toward the M1 type. Gautam et al. [40] upregulated IFN- $\gamma$ to reprogram the phenotype of NLCs to the M1 state and found it may be an effective adjuvant in antibody therapy for CLL. Besides, it was reported that CSF1R was required for NLC survival in CML [41], and inhibitors of CSF1R were similarly demonstrated to have antineoplastic activity through eradication of NLCs [42]. These results establish the important role of macrophages in CLL and suggest therapeutic strategies based on interfering with leukemia-macrophage interactions.

Clinical approaches to the therapeutic targeting of TAMs are under evaluation. As an example, the Bruton tyrosine kinase inhibitor ibrutinib is reported to reduce CLL cell chemoattraction by inhibiting macrophage secretion of CXCL13. Fiorcari et al. [43] recently showed that ibrutinib targeted Bruton tyrosine kinase in NLCs and modified their phenotypes and functions. Treatment with ibrutinib reduced the phagocytic ability and increased the immunosuppressive profile of NLCs exacerbating the expression of M2 markers. Recent studies have shown that NLCs treated with ibrutinib have resistance to chemotherapy-induced apoptosis of CLL cells, which may be related to the secretion of IL-10 [44]. Therefore, the role of ibrutinib in CLL therapy may be reassessed, especially in patients with unsatisfactory outcomes.

Lenalidomide is an immunomodulatory agent clinically active in CLL patients. Fiorcari et al. [45] observed that lenalidomide induced NLCs to lose the ability to nurture CLL cells, acquire properties of phagocytosis, and inhibit the development of CLL. Schulz et al. [46] also observed that lenalidomide reduced the survival support of NLCs for CLL cells in vitro, suggesting that this drug affects the microenvironment in CLL in vivo. Both sets of data support the notion that lenalidomide mediates a proinflammatory switch of NLCs affecting the protective microenvironment generated by CLL.

\section{Conclusions}

Over the last few years, the protective effect of LAMs on leukemia cells has been gradually recognized. LAMs interact with leukemic cells to support leukemia progres- 
sion. However, the molecular mechanisms of LAMs in different types of leukemia cell are diverse because of the distinct microenvironments. Further research in this field may be helpful to establish new therapeutic targets in leukemia.

\section{Disclosure Statement}

The authors declare that they have no competing interests.

\section{Funding Sources}

This work was supported by grant 81670104 from the National Natural Science Foundation of China (NSFC).

\section{Author Contributions}

Y. Li and M.J. You drafted the paper; Y. Yang and D. Hu revised the paper; C. Tian critically revised the paper. All authors approved all versions including the final version and are responsible for the accuracy and integrity of all aspects of the manuscript.

\section{References}

1 Marchesi F, Cirillo M, Bianchi A, Gately M, Olimpieri OM, Cerchiara E, et al. High density of CD68+/CD163+ tumour-associated macrophages (M2-TAM) at diagnosis is significantly correlated to unfavorable prognostic factors and to poor clinical outcomes in patients with diffuse large B-cell lymphoma. Hematol Oncol. 2015 Jun;33(2):110-2.

$2 \mathrm{Hu}$ JM, Liu K, Liu JH, Jiang XL, Wang XL, Yang L, et al. The increased number of tumorassociated macrophage is associated with overexpression of VEGF-C, plays an important role in Kazakh ESCC invasion and metastasis. Exp Mol Pathol. 2017 Feb;102(1):15-21.

3 Liu D, Chang C, Lu N, Wang X, Lu Q, Ren X, et al. Comprehensive proteomics analysis reveals metabolic reprogramming of tumorassociated macrophages stimulated by the tumor microenvironment. J Proteome Res. 2017 Jan;16(1):288-97.

4 Wynn TA, Chawla A, Pollard JW. Macrophage biology in development, homeostasis and disease. Nature. 2013 Apr;496(7446): 445-55.

5 Cai L, Michelakos T, Deshpande V, Arora KS, Yamada T, Ting DT, et al. Role of tumorassociated macrophages in the clinical course of pancreatic neuroendocrine tumors (PanNETs). Clin Cancer Res. 2019 Apr 15;25(8): 2644-55.

6 Grossman JG, Nywening TM, Belt BA, Panni RZ, Krasnick BA, DeNardo DG, et al. Recruitment of CCR2+ tumor associated macrophage to sites of liver metastasis confers a poor prognosis in human colorectal cancer. Oncoimmunology. 2018 Aug;7(9):e1470729.

7 Hanahan D, Coussens LM. Accessories to the crime: functions of cells recruited to the tumor microenvironment. Cancer Cell. 2012 Mar;21(3):309-22.

8 Jafarzadeh N, Safari Z, Pornour M, Amirizadeh N, Forouzandeh Moghadam M, Sadeghizadeh M. Alteration of cellular and immune-related properties of bone marrow mesenchymal stem cells and macrophages by K562 chronic myeloid leukemia cell derived exosomes. J Cell Physiol. 2019 Apr;234(4): 3697-710.
9 Mosser DM, Edwards JP. Exploring the full spectrum of macrophage activation. Nat Rev Immunol. 2008 Dec;8(12):958-69.

10 Gjomarkaj M, Pace E, Melis M, Spatafora M, Profita M, Vignola AM, et al. Phenotypic and functional characterization of normal rat pleural macrophages in comparison with autologous peritoneal and alveolar macrophages. Am J Respir Cell Mol Biol. 1999 Jan; 20(1):135-42.

11 Laskin DL, Weinberger B, Laskin JD. Functional heterogeneity in liver and lung macrophages. J Leukoc Biol. 2001 Aug;70(2):16370.

12 Jarosz-Biej M, Kamińska N, Matuszczak S, Cichoń T, Pamuła-Piłat J, Czapla J, et al. M1like macrophages change tumor blood vessels and microenvironment in murine melanoma. PLoS One. 2018 Jan;13(1):e0191012.

13 Biswas SK, Mantovani A. Macrophage plasticity and interaction with lymphocyte subsets: cancer as a paradigm. Nat Immunol. 2010 Oct;11(10):889-96.

14 Kigerl KA, Gensel JC, Ankeny DP, Alexander JK, Donnelly DJ, Popovich PG. Identification of two distinct macrophage subsets with divergent effects causing either neurotoxicity or regeneration in the injured mouse spinal cord. J Neurosci. 2009 Oct;29(43):13435-44.

15 Sica A, Larghi P, Mancino A, Rubino L, Porta C, Totaro MG, et al. Macrophage polarization in tumour progression. Semin Cancer Biol. 2008 Oct; 18(5):349-55.

16 Schreiber RD, Old LJ, Smyth MJ. Cancer immunoediting: integrating immunity's roles in cancer suppression and promotion. Science. 2011 Mar;331(6024):1565-70.

17 Murray PJ, Allen JE, Biswas SK, Fisher EA, Gilroy DW, Goerdt S, et al. Macrophage activation and polarization: nomenclature and experimental guidelines. Immunity. $2014 \mathrm{Jul}$; 41(1):14-20.

18 Mantovani A, Sozzani S, Locati M, Allavena P, Sica A. Macrophage polarization: tumorassociated macrophages as a paradigm for polarized M2 mononuclear phagocytes. Trends Immunol. 2002 Nov;23(11):549-55.
19 Komohara Y, Jinushi M, Takeya M. Clinical significance of macrophage heterogeneity in human malignant tumors. Cancer Sci. 2014 Jan;105(1):1-8.

20 Kaku Y, Imaoka H, Morimatsu Y, Komohara $\mathrm{Y}$, Ohnishi K, Oda $\mathrm{H}$, et al. Overexpression of CD163, CD204 and CD206 on alveolar macrophages in the lungs of patients with severe chronic obstructive pulmonary disease. PLoS One. 2014 Jan;9(1):e87400.

21 Komohara Y, Hirahara J, Horikawa T, Kawamura K, Kiyota E, Sakashita N, et al. AM-3K, an anti-macrophage antibody, recognizes CD163, a molecule associated with an anti-inflammatory macrophage phenotype. J Histochem Cytochem. 2006 Jul;54(7): 763-71.

22 Laoui D, Movahedi K, Van Overmeire E, Van den Bossche J, Schouppe E, Mommer C, et al. Tumor-associated macrophages in breast cancer: distinct subsets, distinct functions. Int J Dev Biol. 2011;55(7-9):861-7.

23 Chen SY, Yang X, Feng WL, Liao JF, Wang LN, Feng L, et al. Organ-specific microenvironment modifies diverse functional and phenotypic characteristics of leukemia-associated macrophages in mouse $\mathrm{T}$ cell acute lymphoblastic leukemia. J Immunol. 2015 Mar;194(6):2919-29.

24 Chen S, Yang X, Feng W, Yang F, Wang R, Chen $\mathrm{C}$, et al. Characterization of peritoneal leukemia-associated macrophages in Notch1-induced mouse $\mathrm{T}$ cell acute lymphoblastic leukemia. Mol Immunol. 2017 Jan;81:35-41.

25 Yang X, Feng W, Wang R, Yang F, Wang L, Chen S, et al. Hepatic leukemia-associated macrophages exhibit a pro-inflammatory phenotype in Notch1-induced acute T cell leukemia. Immunobiology. 2018 Jan;223(1): 73-80.

26 Komohara Y, Niino D, Saito Y, Ohnishi K, Horlad H, Ohshima K, et al. Clinical significance of $\mathrm{CD} 163^{+}$tumor-associated macrophages in patients with adult T-cell leukemia/ lymphoma. Cancer Sci. 2013 Jul;104(7):94551. 
27 Liao J, Feng W, Wang R, Ma S, Wang L, Yang $\mathrm{X}$, et al. Diverse in vivo effects of soluble and membrane-bound M-CSF on tumor-associated macrophages in lymphoma xenograft model. Oncotarget. 2016 Jan;7(2):1354-66.

28 Komohara Y, Noyori O, Saito Y, Takeya H, Baghdadi M, Kitagawa F, et al. Potential antilymphoma effect of M-CSFR inhibitor in adult T-cell leukemia/lymphoma. J Clin Exp Hematop. 2018;58(4):152-60.

29 Al-Matary YS, Botezatu L, Opalka B, Hönes JM, Lams RF, Thivakaran A, et al. Acute myeloid leukemia cells polarize macrophages towards a leukemia supporting state in a Growth factor independence 1 dependent manner. Haematologica. 2016 Oct;101(10):1216-27.

30 Yang X, Feng W, Wang R, Yang F, Wang L, Chen S, et al. Repolarizing heterogeneous leukemia-associated macrophages with more M1 characteristics eliminates their pro-leukemic effects. Oncoimmunology. 2017 Dec; 7(4):e1412910.

31 Majeti R, Chao MP, Alizadeh AA, Pang WW, Jaiswal S, Gibbs KD Jr, et al. CD47 is an adverse prognostic factor and therapeutic antibody target on human acute myeloid leukemia stem cells. Cell. 2009 Jul;138(2):286-99.

32 Jiang M, Zhang J, Qian L, Miao Y, Song W, Liu $\mathrm{H}$, et al. MOZ forms an autoregulatory feedback loop with miR-223 in AML and monocyte/macrophage development. iScience. 2019 Jan;11:189-204.

33 Burger JA, Tsukada N, Burger M, Zvaifler NJ, Dell'Aquila M, Kipps TJ. Blood-derived nurse-like cells protect chronic lymphocytic leukemia B cells from spontaneous apoptosis through stromal cell-derived factor-1. Blood. 2000 Oct;96(8):2655-63.
34 Tsukada N, Burger JA, Zvaifler NJ, Kipps TJ. Distinctive features of "nurselike" cells that differentiate in the context of chronic lymphocytic leukemia. Blood. 2002 Feb;99(3): 1030-7.

35 Boissard F, Fournié JJ, Laurent C, Poupot M, Ysebaert L. Nurse like cells: chronic lymphocytic leukemia associated macrophages. Leuk Lymphoma. 2015 May;56(5):1570-2.

36 Boissard F, Laurent C, Ramsay AG, QuilletMary A, Fournié JJ, Poupot M, et al. Nurselike cells impact on disease progression in chronic lymphocytic leukemia. Blood Cancer J. 2016 Jan;6(1):e381.

37 Giannoni P, Pietra G, Travaini G, Quarto R, Shyti G, Benelli R, et al. Chronic lymphocytic leukemia nurse-like cells express hepatocyte growth factor receptor (c-MET) and indoleamine 2,3-dioxygenase and display features of immunosuppressive type 2 skewed macrophages. Haematologica. 2014 Jun;99(6): 1078-87.

38 Hanna BS, McClanahan F, Yazdanparast $\mathrm{H}$, Zaborsky N, Kalter V, Rößner PM, et al. Depletion of CLL-associated patrolling monocytes and macrophages controls disease development and repairs immune dysfunction in vivo. Leukemia. 2016 Mar;30(3):570-9.

39 Galletti G, Scielzo C, Barbaglio F, Rodriguez TV, Riba M, Lazarevic D, et al. Targeting macrophages sensitizes chronic lymphocytic leukemia to apoptosis and inhibits disease progression. Cell Rep. 2016 Feb;14(7):1748-60.
40 Gautam S, Fatehchand K, Elavazhagan S, Reader BF, Ren L, Mo X, et al. Reprogramming nurse-like cells with interferon $\gamma$ to interrupt chronic lymphocytic leukemia cell survival. J Biol Chem. 2016 Jul;291(27): 14356-62.

41 Polk A, Lu Y, Wang T, Seymour E, Bailey NG, Singer JW, et al. Colony-stimulating factor-1 receptor is required for Nurse-like cell survival in chronic lymphocytic leukemia. Clin Cancer Res. 2016 Dec;22(24):6118-28.

42 Edwards V DK, Sweeney DT, Ho H, Eide CA, Rofelty A, Agarwal A, et al. Targeting of colony-stimulating factor 1 receptor (CSF1R) in the CLL microenvironment yields antineoplastic activity in primary patient samples. Oncotarget. 2018 May;9(37):24576-89.

43 Fiorcari S, Maffei R, Audrito V, Martinelli S, Ten Hacken E, Zucchini P, et al. Ibrutinib modifies the function of monocyte/macrophage population in chronic lymphocytic leukemia. Oncotarget. 2016 Oct;7(40):65968-81.

44 Long M, Beckwith K, Do P, Mundy BL, Gordon A, Lehman AM, et al. Ibrutinib treatment improves $\mathrm{T}$ cell number and function in CLL patients. J Clin Invest. 2017 Aug;127(8):305264.

45 Fiorcari S, Martinelli S, Bulgarelli J, Audrito V, Zucchini P, Colaci E, et al. Lenalidomide interferes with tumor-promoting properties of nurse-like cells in chronic lymphocytic leukemia. Haematologica. 2015 Feb;100(2):25362.

46 Schulz A, Dürr C, Zenz T, Döhner H, Stilgenbauer S, Lichter P, et al. Lenalidomide reduces survival of chronic lymphocytic leukemia cells in primary cocultures by altering the myeloid microenvironment. Blood. 2013 Mar; 121(13):2503-11. 